\title{
Pulmonary Sequelae at 4 Months After COVID-19 Infection: A Single-Centre Experience of a COVID Follow-Up Service
}

\author{
Rebecca C. Robey (D) - Katie Kemp · Philip Hayton · Dalia Mudawi • \\ Ran Wang · Melanie Greaves • Veronica Yioe · Pilar Rivera-Ortega • \\ Cristina Avram • Nazia Chaudhuri
}

Received: May 10, 2021 / Accepted: June 17, 2021 / Published online: July 18, 2021

(C) The Author(s) 2021

\section{ABSTRACT}

Introduction: At the end of the first year of the COVID-19 pandemic, more than 78 million known survivors were recorded. The long-term pulmonary sequelae of COVID-19 remain unknown.

Methods: We performed a retrospective analysis of a post-COVID follow-up service to

Supplementary Information The online version contains supplementary material available at https:// doi.org/10.1007/s12325-021-01833-4.

R. C. Robey $(\bowtie) \cdot$ K. Kemp · P. Hayton ·

D. Mudawi · R. Wang · V. Yioe · P. Rivera-Ortega

C. Avram · N. Chaudhuri

Department of Respiratory Medicine, Wythenshawe

Hospital, Manchester University NHS Foundation

Trust, Manchester, UK

e-mail: rebecca.robey@doctors.org.uk

R. C. Robey · R. Wang

Division of Infection, Immunity and Respiratory Medicine, School of Biological Sciences, University of Manchester, Manchester, UK

R. Wang

Manchester Academic Health Science Centre and NIHR Manchester Biomedical Research Centre, Manchester University NHS Foundation Trust, Manchester, UK

\section{Greaves}

Department of Radiology, Wythenshawe Hospital, Manchester University NHS Foundation Trust, Manchester, UK estimate the burden of persistent pulmonary morbidity in hospitalised COVID survivors.

Results: A total of 221 patients were followedup: 44 intensive care unit (ICU) and 177 ward patients. Further investigations were planned as per British Thoracic Society Guidelines: For all ICU patients $(n=44)$ and for 38 of $177(21 \%)$ ward-based patients who had persistent symptoms and/or persistent radiographic changes on CXR at their initial 8-week follow-up visit. In the ward-based cohort, statistically significant associations with persistent symptoms were being an ex- or current smoker, having pre-existing diabetes, and having a longer length of stay. In patients requiring further investigations, pulmonary function tests (PFTs; $n=67$ ) at an average of 15 weeks post-discharge showed abnormalities in at least one PFT parameter in $79 \%$ (equating to $24 \%$ of the entire cohort). The most common abnormality was an abnormal diffusion capacity of carbon monoxide (TLCO), highest in the ICU cohort (64\% ICU vs. $38 \%$ non-ICU). TLCO correlated negatively with length of stay and with maximum inspired $\mathrm{FiO}_{2}$ in the patient group as a whole. In ICU patients, TLCO correlated negatively with maximum inspired positive airway pressure. Computed tomography scans $(n=72)$ at an average of 18 weeks post-discharge showed evidence of persistent ground glass opacities in $44 \%$ and fibrosis in $21 \%$ (equating to $7 \%$ of the entire cohort). 
Conclusion: Our data add to the growing evidence that there will be pulmonary sequelae in a proportion of COVID survivors, providing some insight into what may become a significant chronic global health problem.

Keywords: COVID-19;

Coronavirus; Pulmonary sequelae; Pulmonary function tests; Radiological fibrosis

\section{Key Summary Points}

Why carry out this study?

The long-term effects of COVID on the lungs remain unclear, but, given the extent of the pandemic, it has the potential to become a significant chronic global health problem.

New health services need to be rolled out to follow-up COVID survivors, and understanding what these might entail is helpful for future planning.

We provide the first description of findings from a COVID follow-up service designed in accordance with British Thoracic Society Guidelines

\section{What was learned from this study?}

A total of 221 patients were followed-up: all patients who had been admitted to ICU $(n=44)$ and 38 of 177 (21\%) non-ICU patients who had persistent symptoms and/or persistent radiographic changes on chest X-ray at their initial 8-week followup visit required further pulmonary function testing and thoracic imaging.

An overall prevalence of PFT abnormalities were seen in around $24 \%$ of the entire cohort and radiological evidence of fibrosis was seen in around $7 \%$ of the entire cohort at 4 months after discharge.

\section{INTRODUCTION}

On 31 December 2019, the Wuhan Municipal Health Commission, China, reported a cluster of cases of pneumonia of unknown origin to the World Health Organisation (WHO) [1]. The causative agent was a novel coronavirus SARSCoV-2, now widely known as COVID-19. A year later, on 30 December 2020, the WHO reported a total number of $80,453,105$ confirmed cases globally, and 1,775,776 confirmed deaths globally [2].

There is very little knowledge of the incidence of long-term sequelae following COVID19 [3]. Although COVID-19 is a systemic disease, evidence suggests that the lungs are the most affected organ [4]. Follow-up data from previous coronavirus outbreaks, SARS-CoV and MERS-CoV, indicate that between 25\% and 35\% of survivors experience persistent abnormalities of pulmonary function and radiographic changes consistent with pulmonary fibrosis $[5,6]$. Early case reports indicate that this phenomenon will also be associated with postCOVID infection [7-10]. With a global population of more than 78 million COVID-19 survivors at the close of 2020, and case rates continuing to rise, a better understanding of what may become a significant chronic global health issue is essential.

A handful of studies have described pulmonary sequelae of COVID in a total of 1509 post-infection COVID patients (individual study sample sizes 18-246 patients). Follow-up time ranged from 2 weeks to 3 months. Most studies report persistent radiographic changes and abnormalities of pulmonary function tests (PFTs) in a proportion of their patients, with prevalence varying from 18 to $90 \%$ for computerised tomography (CT) changes and $25 \%$ to $88 \%$ for PFT abnormalities [11-27].

In this study, we report on the experience of a newly established COVID follow-up service. We aimed to add to the growing evidence of the long-term effects of COVID-19, and to make early estimates of the persistence of pulmonary morbidity in hospitalised COVID survivors, and therefore the potential future burden to health services. Our objectives were to ascertain: (1) 
the proportion of hospitalised COVID-19 survivors requiring follow-up investigations (PFTs and CT imaging) in accordance with British Thoracic Society (BTS) guidelines [28]; (2) factors associated with this need for follow-up; and (3) the prevalence of persistent PFT abnormalities and CT changes in those requiring followup investigations. Our findings will help inform the design and roll-out of services in the postCOVID era.

\section{METHODS}

\section{Study Subjects and Design}

This was a retrospective service evaluation analysis of the post-COVID follow-up service established at Manchester University NHS Foundation Trust, Wythenshawe Hospital, a tertiary respiratory department in the United Kingdom, in the wake of the first wave of the COVID-19 pandemic in spring 2020.

All consecutive patients admitted with a diagnosis of COVID-19 under Respiratory Medicine at Wythenshawe Hospital between 1 March and 30 June 2020 were included $(n=283)$. Diagnosis was made either by a positive SARS-CoV-2 reverse-transcriptase polymerase chain reaction or a combination of high clinical suspicion and supportive imaging, at the discretion of a senior clinician. Fifty-three of these individuals died (19\%) during admission, while nine declined follow up. The remaining 221 patients were referred for follow-up. Followup PFTs and CT thoracic imaging were planned for patients as per BTS guidelines [28].

\section{Ethics Statement}

The study was conducted according to the guidelines of the Declaration of Helsinki of 1964, and its later amendments. Ethical review and approval were waived for this study, due to the work conducted being part of local service evaluation, utilising only data routinely collected as part of the local COVID follow-up service. As such, specific ethical approval and informed patient consent were not required, as confirmed by the NHS Health Research Authority decision tool. This service evaluation is registered locally.

\section{Methods}

Patient demographic data, including age, ethnicity, gender, pre-admission body mass index (BMI) and pre-existing co-morbidities, were obtained from hospital electronic patient records (EPR) and electronic general practice records, where available. Need for supplemental oxygen, admission to the intensive care unit (ICU), length of hospital stay, maximum fraction of inspired oxygen $\left(\mathrm{FiO}_{2}\right)$ required, peak positive end-expiratory pressure (PEEP) and peak inspiratory positive airway pressure (IPAP) for ventilated patients were obtained via EPR and ICU notes.

All patients admitted under respiratory medicine or ICU were assessed by telephone at approximately 8 weeks post-discharge. A standard follow-up proforma (supplementary material 1) was used to identify persistent symptoms, including cough, breathlessness and fatigue. Breathlessness both pre- and post-admission was recorded and graded as per the Medical Research Council (MRC) breathlessness score. Follow-up chest radiographs were offered to all patients, and were reported by the local radiology department as per the British Society of Thoracic Imaging guidelines [29]. Patients were either discharged from the follow-up service, or further investigated with pulmonary function testing and high-resolution computed tomography thorax. Reasons for further investigation were: persistent breathlessness from baseline (MRC breathlessness score increase of $\geq 1$ ), significantly reduced exercise tolerance (self-reported), or persistent or worsening chest radiograph changes at follow-up. All CT scans were reported by a specialist thoracic radiologist with expertise in interstitial lung disease. Based on the descriptive report, the changes were categorized as: (1) normal; (2) persistent ground glass opacification; or (3) features of fibrosis. 


\section{Analysis}

Main outcome measures:

1. Factors associated with persistent symptoms and/or changes on chest radiograph at 8 weeks post-discharge.

2. Pulmonary function tests at 4 months postdischarge in discharged ICU patients and ward-based patients with persistent symptoms at 8 weeks.

3. Radiographic changes on CT thorax at 4 months post-discharge in ICU patients and ward patients with persistent symptoms.

Statistical analysis was performed using Microsoft Excel and GraphPad Prism 9 software. Categorical variables were presented as counts, whilst continuous variables were presented as means \pm standard deviation (SD). Statistical significance was tested using the Student's $t$ test, Fisher's exact test, chi-squared test, Mann-Whitney test, or one-way ANOVA test, as appropriate. Correlation was tested using Spearman's correlation coefficient. A $p$ value of $<0.05$ is denoted as statistically significant.

\section{RESULTS}

\section{Baseline Demographics (Table 1)}

A total of 221 patients were included for analysis, of which 135 (61\%) were male. Average age at admission was 58 years and average pre-admission BMI was $30.0 \mathrm{~kg} / \mathrm{m}^{2}$. The majority of patients were white $(n=172,78 \%), 31$ were Asian (14\%), 9 were black (4\%) and 9 were of another ethnicity (4\%). The majority of patients were life-long non-smokers $(n=139,63 \%)$. The most common pre-existing co-morbidity was hypertension $(n=74,33 \%)$, followed by asthma $(n=43,19 \%)$, diabetes mellitus (DM; $\mathrm{n}=36,16 \%)$, chronic obstructive pulmonary disease (COPD; $n=19,9 \%$ ) and cardiovascular disease $(n=19,9 \%)$. Nearly a quarter of patients $(n=53,24 \%)$ had two or more pre-existing comorbidities (Table 1).

Forty-four patients were treated in the ICU. The average age of ICU patients was younger than non-ICU patients (54 vs. 59 years, $p=0.033)$. The prevalence of COPD was increased in the non-ICU cohort compared with the ICU cohort $(11 \%$ vs. $0 \%, p=0.016)$. This is likely due to pre-existing COPD being felt to represent a poor prognostic factor with respect to the likelihood of benefit and survival in ICU, therefore reducing the likelihood of escalation of affected patients beyond ward-level care. There were no other statistically significant differences between the two cohorts (Table 1).

We compared baseline demographics of nonICU patients with persistent symptoms and/or radiographic changes at 8 weeks (and therefore required further follow-up) and those that were symptom-free at 8 weeks. A greater proportion of patients with persistent symptoms were exor current smokers compared to those who were symptom-free with normal chest X-rays (CXRs) at 8 weeks $(53 \%$ vs. $34 \%, p=0.040)$ (see Table 1). Pre-existing DM was also statistically significantly increased in those requiring further follow-up compared with those discharged at 8 weeks ( $29 \%$ vs. $10 \%, p=0.007)$. There was a trend towards increased prevalence of COPD in the follow-up group (18\% vs. $9 \%$ ) but this did not reach statistical significance $(p=0.134)$ (Table 1). There was no association between preexisting asthma and the need for further followup (Table 1).

\section{Inhaled Corticosteroids and Oral Corticosteroids in Concurrent Airways Disease}

Pre-admission inhaled corticosteroids (ICS) were prescribed in all 19 patients with a pre-existing diagnosis of COPD or asthma-COPD overlap syndrome, and in 33 of 40 (83\%) patients with a preexisting diagnosis of asthma. There was no difference between the rates of prescribed ICS between the ICU and non-ICU cohort (25\% vs. 23\%; $p=0.695$ ) (Table 1 ). There was also no significant difference between rates of prescribed ICS between the follow-up (FU) and non-FU groups in the nonICU cohort (34\% vs. $19 \% ; p=0.078)$ (Table 1$)$. Twelve of the patients in the present study received oral prednisolone to treat their concurrent airways disease during admission for COVID, but, given 


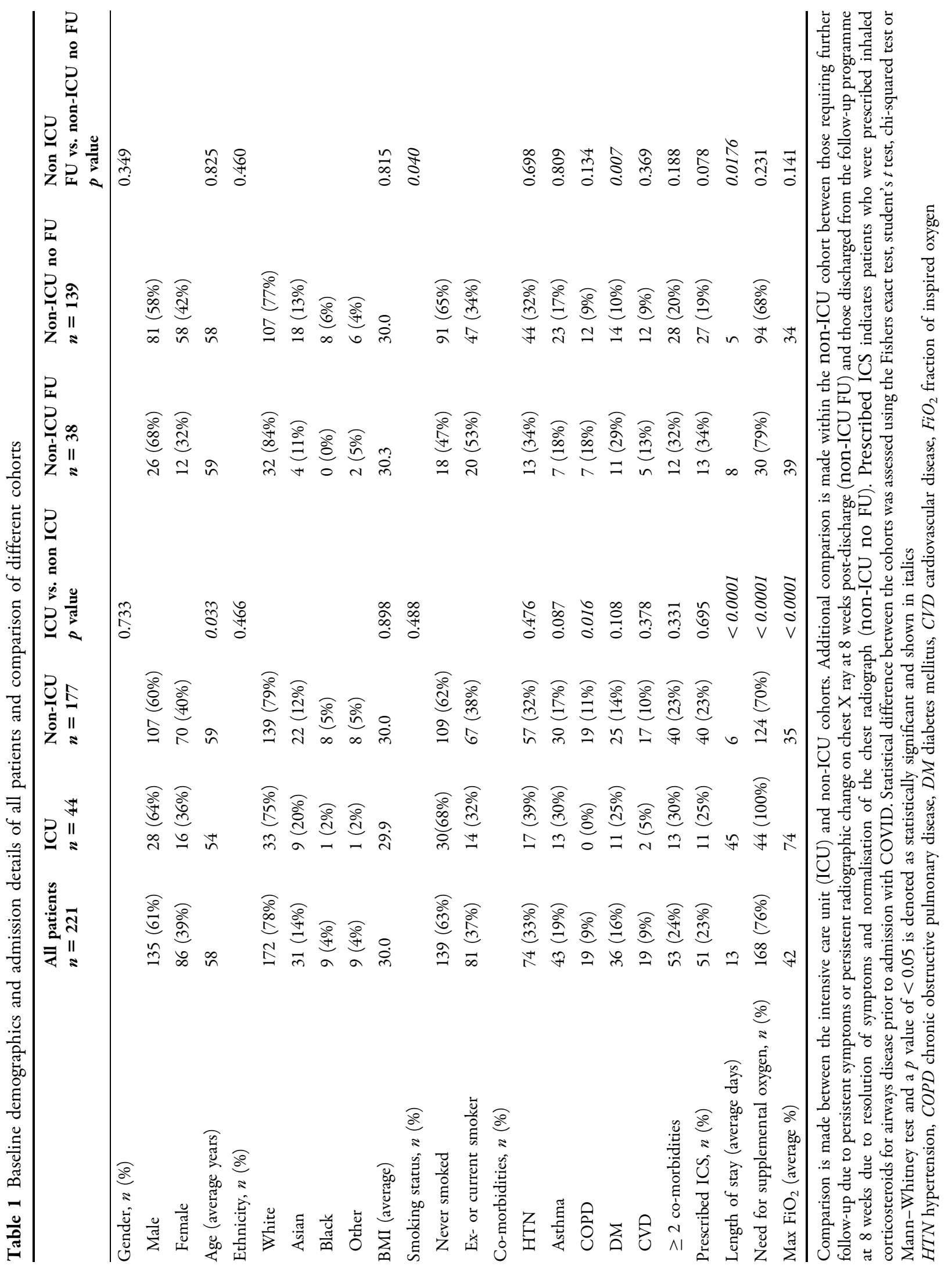




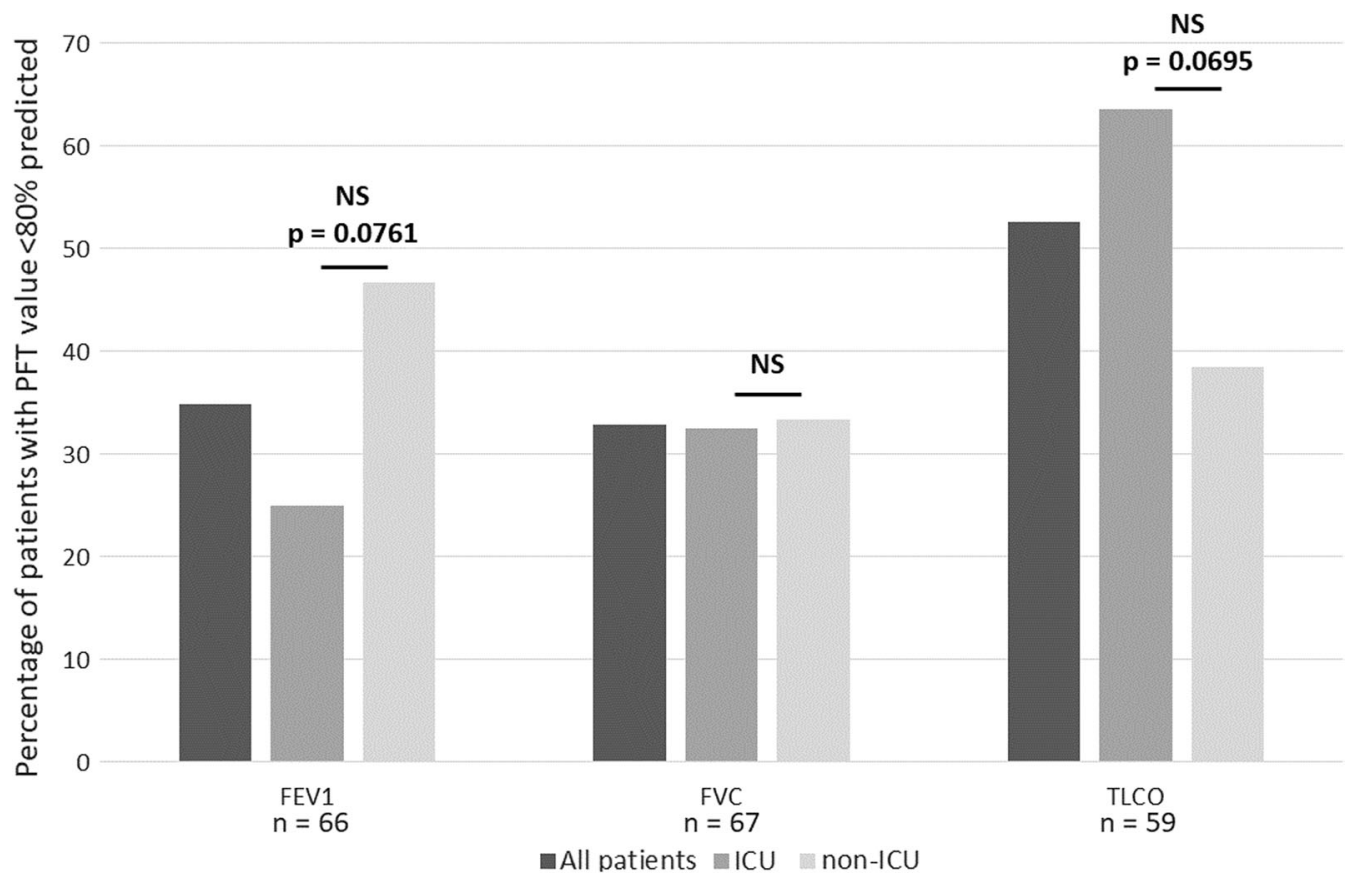

Fig. 1 The proportion of patients with abnormal pulmonary function tests comparing ICU and non-ICU cohorts. Abnormal pulmonary function tests (PFT) are defined as $<80 \%$ predicted value for FEV1, FVC, and TLCO. Comparison is made between intensive care unit (ICU) patients and those non-ICU patients who required follow-up investigations. Not all PFTs were

the small numbers involved, we were not able to draw any conclusions regarding the impact of this treatment (data not shown).

\section{Hospital Length of Stay and Oxygen Therapy}

The ICU cohort had a longer length of stay (LOS), a greater need for supplemental oxygen, and a higher maximum $\mathrm{FiO}_{2}$ than the non-ICU cohort (Table 1). LOS was also significantly different between the non-ICU group requiring follow-up compared to those discharged from follow-up at 8 weeks (8 days vs. 5 days; $p=0.0176$ ) (Table 1). There was no difference between the number of patients requiring supplemental oxygen or the maximum $\mathrm{FiO}_{2}$ in the non-ICU group requiring follow-up compared to those discharged from follow-up at 8 weeks (Table 1). completed for all patients. The number of patients completing each test is shown under the appropriate bars. Statistical significance is measured using Fisher's exact test and a $\mathrm{p}$ value of $<0.05$ is denoted as statistically significant. $N S$ not significant, $F E V I$ forced expiratory volume in $1 \mathrm{~s}, F V C$ forced vital capacity, TLCO diffusion capacity for carbon monoxide

\section{Follow-Up Investigations}

Follow-up PFTs and CT thoracic imaging were planned for all ICU patients $(n=44)$ and 38 of 177 (21\%) non-ICU patients who had persistent symptoms and/or persistent radiographic changes on CXR at their initial 8-week followup visit, as per BTS guidelines. Missing data due to patients failing to attend or inability to complete tests is depicted in tables and figures where appropriate.

\section{Abnormalities in Pulmonary Function Tests Correlate with Length of Stay and Maximum $\mathrm{FiO}_{2}$}

Sixty-seven (30\%) patients had follow-up PFTs: 37 of $44(84 \%)$ ICU patients and 30 of 38 nonICU patients intended for follow-up. Pre-admission PFTs were only available for 3 of these 
Table 2 Pulmonary function tests (PFTs) values comparing ICU and non-ICU cohorts

\begin{tabular}{|c|c|c|c|c|}
\hline PFT & $\begin{array}{l}\text { All patients } \\
n=82\end{array}$ & $\begin{array}{l}\text { ICU } \\
n=44\end{array}$ & $\begin{array}{l}\text { Non-ICU } \\
n=38\end{array}$ & $\begin{array}{l}\text { ICU vs. non ICU } \\
p \text { value (Student } T \text { test) }\end{array}$ \\
\hline \multicolumn{5}{|l|}{ FEV1 } \\
\hline$n(\%)$ & $66(80)$ & $36(82)$ & $30(79)$ & \\
\hline$\%$ predicted: mean & 84.7 & 87.7 & 81.0 & 0.107 \\
\hline SD & 17.0 & 16.8 & 16.9 & \\
\hline Absolute value: mean & 2.69 & 2.88 & 2.45 & 0.027 \\
\hline SD & 0.80 & 0.87 & 0.64 & \\
\hline \multicolumn{5}{|l|}{ FVC } \\
\hline$n(\%)$ & $67(82)$ & $37(84)$ & $30(79)$ & \\
\hline$\%$ predicted: mean & 86.5 & 86.6 & 86.2 & 0.922 \\
\hline SD & 16.6 & 17.9 & 15.2 & \\
\hline Absolute value: mean & 3.46 & 3.59 & 3.31 & 0.255 \\
\hline SD & 0.98 & 1.14 & 0.71 & \\
\hline \multicolumn{5}{|l|}{ FEV1/FVC } \\
\hline$n(\%)$ & $65(79)$ & $35(80)$ & $30(79)$ & \\
\hline Mean & 77.72 & 80.91 & 74.00 & 0.003 \\
\hline SD & 9.66 & 6.92 & 14.28 & \\
\hline \multicolumn{5}{|l|}{ MFEF $75 / 25$} \\
\hline$n(\%)$ & $65(79)$ & $35(80)$ & $30(79)$ & \\
\hline$\%$ predicted: mean & 92.1 & 102.1 & 80.4 & 0.022 \\
\hline SD & 38.3 & 33.5 & 40.8 & \\
\hline Absolute value: mean & 2.61 & 2.99 & 2.17 & 0.004 \\
\hline SD & 1.17 & 1.08 & 1.12 & \\
\hline \multicolumn{5}{|l|}{ TLCO } \\
\hline$n(\%)$ & $59(72)$ & $33(75)$ & $26(68)$ & \\
\hline$\%$ predicted: mean & 76.6 & 74.0 & 79.9 & 0.275 \\
\hline SD & 20.6 & 20.5 & 20.7 & \\
\hline Absolute value: mean & 6.55 & 6.47 & 6.66 & 0.751 \\
\hline SD & 2.23 & 2.37 & 2.09 & \\
\hline
\end{tabular}

Comparison is made between intensive care unit (ICU) patients and non-ICU patients. Not all PFTs were completed for all patients. The number of patients completing each test is in the appropriate cell. Statistical significance is measured using Student's $t$ test and a $p$ value of $<0.05$ is denoted as statistically significant and shown in italics FEVI forced expiratory volume in one second, FVC forced vital capacity, MFEF 75/25 forced expiratory flow 25-75\%, $T L C O$ diffusion capacity for carbon monoxide 

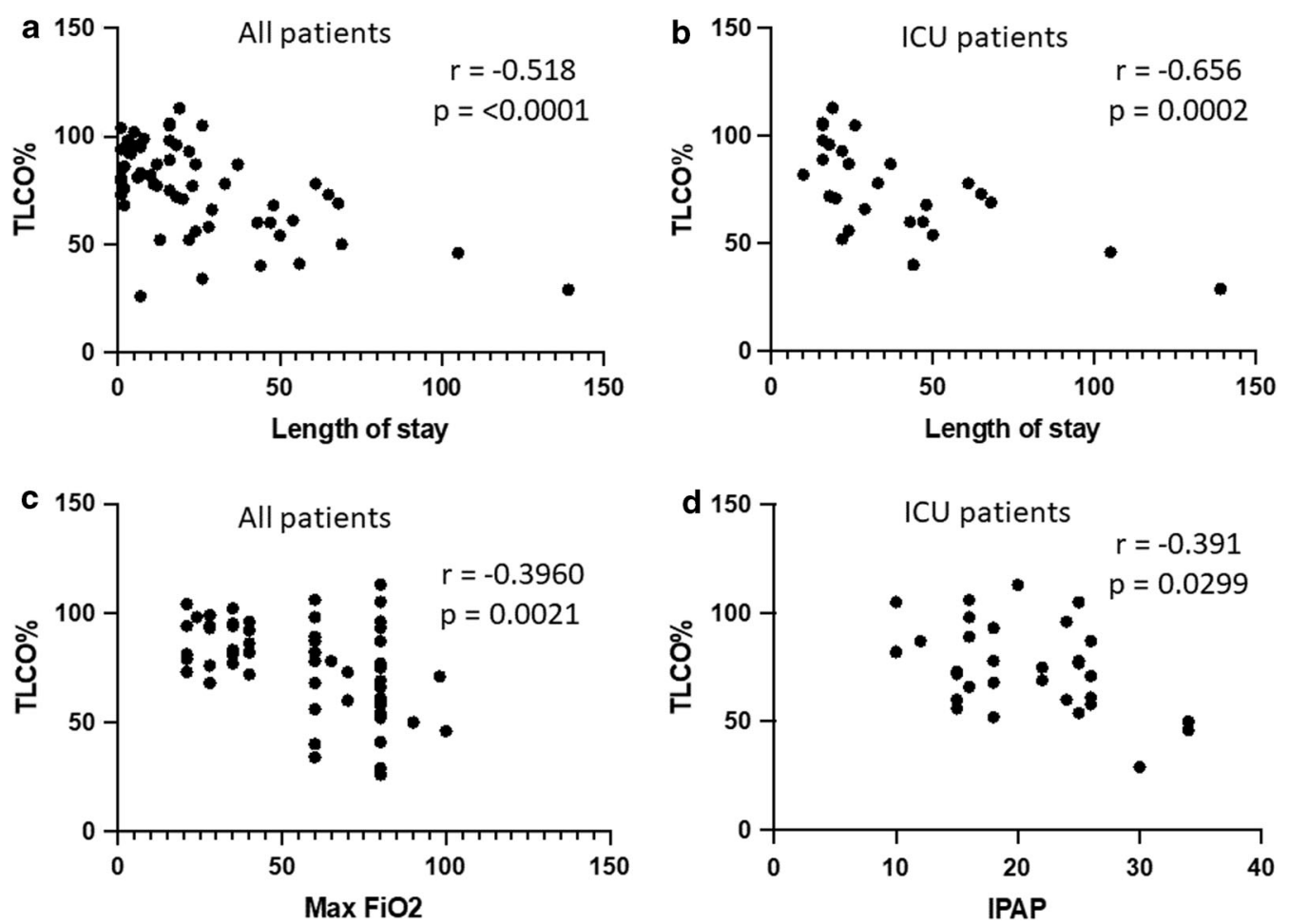

Fig. 2 Percentage of predicted TLCO correlates negatively with increasing length of stay, increasing maximum $\mathrm{FiO}_{2}$ and peak inspiratory positive airway pressure (IPAP). a Percentage of predicted TLCO compared with length of stay for all patients. b Percentage of predicted TLCO compared with length of stay for intensive care unit (ICU) patients. c Percentage of predicted TLCO compared with

individuals (data not shown). The average time between discharge date and follow-up PFTs was $109 \pm 37$ days (median $=101)$. Fifty-three of the $67(79 \%)$ had an abnormality $(<80 \%$ predicted value) in at least one PFT parameter. This equated to $24 \%$ of the entire cohort $(n=221)$. There was no difference in the proportion of patients with at least one abnormal PFT between the ICU and non-ICU groups (76\% vs. $83 \% ; p=0.552$ ).

The most common pulmonary function abnormality was a reduction in diffusion capacity of carbon monoxide (TLCO) of less than $80 \%$ predicted, seen in 31 of 59 patients (53\%). This was more frequent in ICU patients compared with non-ICU patients, but did not reach statistical significance $(64 \%$ vs. $38 \%$; $p=0.0695$ ) (Fig. 1). A restrictive deficit [forced vital capacity (FVC) $<80 \%$ predicted and forced

maximum inspired $\mathrm{FiO}_{2}$ for all patients. d Peak IPAP correlates with percentage predicted TLCO in ventilated patients on ICU. Correlation is tested using Spearman's correlation coefficient, and a $p$ value of $<0.05$ is denoted as statistically significant. TLCO diffusion capacity for carbon monoxide

expiratory volume in one second/forced vital capacity (FEV1/FVC) ratio $>70 \%]$ was seen in 22 of 67 patients (33\%), and was similarly distributed in ICU and non-ICU patients. There was no statistical difference in absolute and percentage predicted FVC and TLCO values between the ICU and non-ICU groups (Table 2).

The non-ICU cohort had a significantly lower absolute FEV1 (2.45 vs. 2.88; $p=0.027$ ) and absolute and percentage predicted MFEF $75 / 25$ ( 2.17 vs. $2.99 ; p=0.027$, and 80 vs. 102 ; $p=0.004$, respectively) than the ICU cohort (Table 2). We speculate that the increased prevalence of obstructive airway disease and small airway disease in the non-ICU follow-up group compared to the ICU group is likely explained by the increased prevalence of COPD (18\% vs. $0 \%)$ and ex- or current smokers (53\% vs. $32 \%$ ) in this group (Fig. 1). 


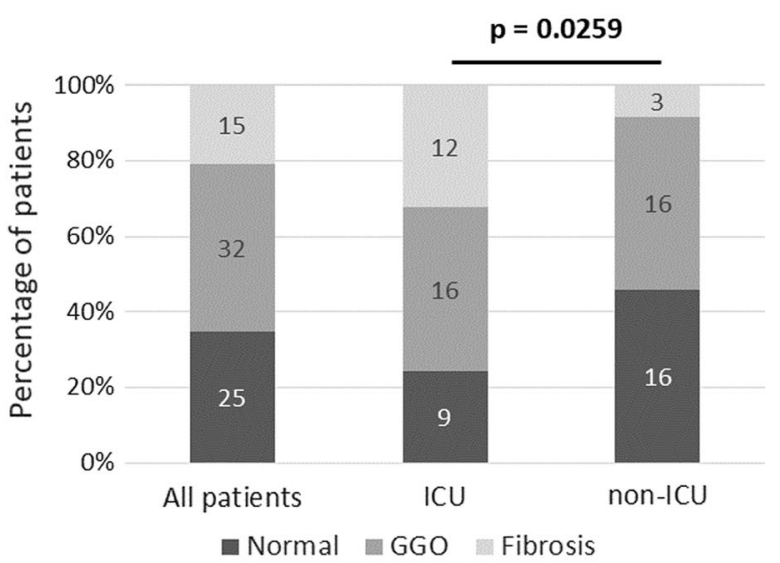

Fig. 3 Radiological findings on CT Thorax at an average of 5 months after admission. a Prevalence of ground glass changes (GGO) and fibrotic changes on CT thorax at follow up. Comparison is made between intensive care unit (ICU) patients and those non-ICU patients who required follow-up investigations. These changes were significantly increased in intensive care unit (ICU) patients as compared to non-ICU patients (chi-squared test).

For the cohort as a whole, longer LOS correlated with declining TLCO $(r=-0.518$; $p=<0.0001)$. This remained true for the ICU cohort $(r=-0.656 ; p=0.0002)$ but not the non-ICU cohort $(r=-0.286 ; \mathrm{p}=0.153)$ when analysed separately (Fig. 2a, b). For the cohort as a whole, higher maximum $\mathrm{FiO}_{2}$ correlated with declining TLCO $\quad(r=-0.396 ; p=0.0021)$ (Fig. 2c). When analysed separately, this correlation was not maintained in either the ICU cohort $(r=-0.0986 ; p=0.624)$ or the non-ICU cohort $(r=-0.324 ; p=0.1136)$. In the ICU cohort, higher maximum IPAP correlated with declining TLCO $(r=-0.391 ; p=0.0299)$ (Fig. $2 \mathrm{~d}$ ) but not PEEP ( $r=0.083 ; p=0.693)$. In all patients, there was no correlation between TLCO and BMI $(r=0.0404 ; p=0.765)$.

\section{Thoracic Radiology}

A total of $72(33 \%)$ follow-up CT scans were performed: 37 of $44(84 \%)$ ICU patients and 35 of $38(92 \%)$ non-ICU patients intended for follow-up. The mean time from discharge to follow-up CT imaging was $131 \pm 52$ days (median $=132$ ). For those who had acute imaging from their admission with COVID, all

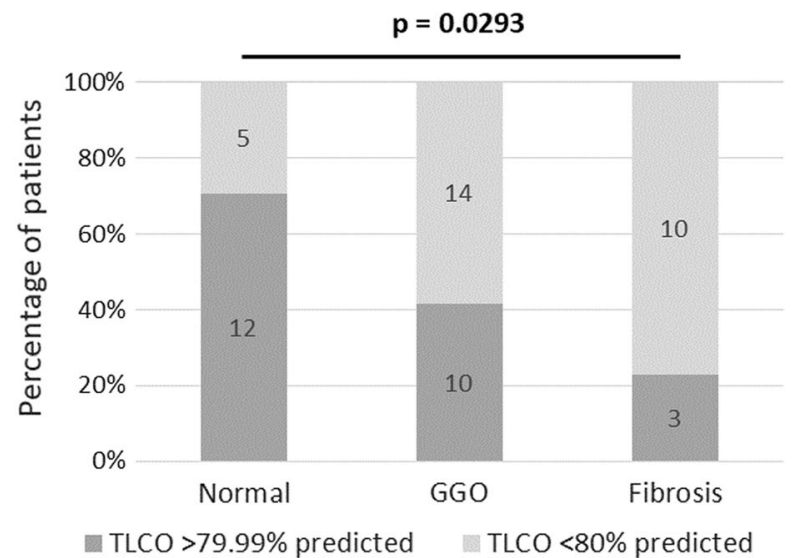

b Prevalence of abnormalities in TLCO $(<80 \%$ predicted value) in each of the three radiological groups. These were significantly increased in those with ground glass changes and fibrotic change as compared to those with normal appearance on CT. Statistical significance is measured using the chi-squared test and a $p$ value of $<0.05$ is denoted as statistically significant

were reported to show some temporal improvement in relation to the admission CT. Figure 3a shows the proportion of CT scans that were defined as normal (35\%), ground-glass opacities (GGO; 44\%), or had radiological signs of fibrosis (defined as evidence of traction bronchiectasis, architectural distortion, or honey combing; $21 \%$ ). This equated to GGO in $14 \%$ and fibrosis in $7 \%$ of the entire cohort $(n=221)$. Radiological changes had a statistically significant increase in the ICU compared with the ward-based cohorts (chi-squared test, $p=0.0259$ ): GGO in $43 \%$ ICU compared with $46 \%$ non-ICU, and fibrosis in $32 \%$ ICU compared with 9\% non-ICU. TLCO abnormalities were more frequent in those with GGO or fibrosis compared to those with normal appearances (chi-squared test, $p=0.0293$ ) (Fig. 3b).

Maximum inspired $\mathrm{FiO}_{2}$ was highest in the fibrosis group (mean $=74.3$ ), followed by those with GGO (mean =56.6) and lowest in the normal CT imaging group (mean $=47.4$ ), and this trend reached statistical significance (oneway ANOVA test, $p=0.0038$ ) (Table 3). There were no differences in peak PEEP or IPAP between the three groups. There was a 
Table 3 Maximum inspired $\mathrm{FiO}_{2}$, ventilator settings and percentage of predicted values for pulmonary function tests (PFTs) in the different radiological categories

\begin{tabular}{|c|c|c|c|c|}
\hline & $\begin{array}{l}\text { Normal } \\
n=25 \text { (all) } \\
n=9 \text { (ICU) }\end{array}$ & $\begin{array}{l}\text { Ground glass } \\
\begin{array}{l}n=32 \text { (all) } \\
n=16(\text { ICU })\end{array}\end{array}$ & $\begin{array}{l}\text { Fibrosis } \\
n=15 \text { (all) } \\
n=12 \text { (ICU) }\end{array}$ & $p$ value (one-way ANOVA) \\
\hline \multicolumn{5}{|l|}{$\mathrm{Max} \mathrm{FiO}_{2}$ (all) } \\
\hline$n(\%)$ & $25(100)$ & $31(97)$ & $15(100)$ & \\
\hline Mean & 47.4 & 56.6 & 74.3 & 0.0038 \\
\hline SD & 21.4 & 23.5 & 20.7 & \\
\hline \multicolumn{5}{|l|}{ Peak PEEP (ICU) } \\
\hline$n(\%)$ & $8(89)$ & $16(100)$ & $11(92)$ & \\
\hline Mean & 12.4 & 12.2 & 12.7 & 0.845 \\
\hline SD & 3.5 & 2.1 & 1.6 & \\
\hline \multicolumn{5}{|l|}{ Peak IPAP (ICU) } \\
\hline$n(\%)$ & $8(89)$ & $16(100)$ & $11(92)$ & \\
\hline Mean & 19.6 & 19.7 & 24.1 & 0.182 \\
\hline SD & 6.5 & 5.7 & 7.3 & \\
\hline \multicolumn{5}{|l|}{ FEV1 (all) } \\
\hline$n(\%)$ & $18(72)$ & $29(91)$ & $13(87)$ & \\
\hline$\%$ predicted: mean & 87.4 & 83.1 & 86.2 & 0.696 \\
\hline SD & 13.7 & 19.8 & 16.8 & \\
\hline Absolute value: mean & 2.77 & 2.64 & 2.80 & 0.790 \\
\hline SD & 0.60 & 0.90 & 0.79 & \\
\hline \multicolumn{5}{|l|}{ FVC (all) } \\
\hline$n(\%)$ & $18(72)$ & $30(94)$ & $13(87)$ & \\
\hline$\%$ predicted: mean & 92.0 & 85.1 & 83.6 & 0.305 \\
\hline SD & 12.8 & 18.4 & 18.0 & \\
\hline Absolute value: mean & 3.68 & 3.41 & 3.48 & 0.664 \\
\hline SD & 0.63 & 1.10 & 1.03 & \\
\hline \multicolumn{5}{|l|}{ TLCO (all) } \\
\hline$n(\%)$ & $17(68)$ & $24(75)$ & $13(87)$ & \\
\hline$\%$ predicted: mean & 87.1 & 75.2 & 64.5 & 0.007 \\
\hline SD & 10.3 & 22.7 & 19.1 & \\
\hline Absolute value: mean & 7.59 & 6.50 & 5.56 & 0.041 \\
\hline SD & 1.57 & 2.42 & 2.19 & \\
\hline
\end{tabular}

Comparison is made between those with normal, ground glass opacities (GGO), and fibrosis on CT imaging. Maximum ventilator settings (PEEP) and (IPAP) are shown for ICU patients. Not all PFTs were completed for all patients, the number of patients completing each test is indicated. Statistical significance is measured using a one-way ANOVA test; with a $p$ value $<0.05$ as statistically significant and shown in italics

FEVI forced expiratory volume in $1 \mathrm{~s}, F V C$ forced vital capacity, TLCO diffusion capacity for carbon monoxide 
statistically significant difference in TLCO between the three groups (one-way ANOVA test, $p=0.0072$ ), with lowest average TLCO in those showing fibrotic changes (Table 3 ).

\section{DISCUSSION}

We have presented findings from a retrospective evaluation of a 'real-world' experience of following-up hospitalised COVID survivors. We found that, in patients who had received wardlevel care only, $21 \%$ had persistent symptoms or CXR changes at 8 weeks, warranting further investigation in accordance with BTS guidelines. In this cohort, statistically significant associations with persistent symptoms were being an ex- or current smoker, having pre-existing DM, and having a longer length of stay. To the best of our knowledge, we are the first to report these associations. The association with $\mathrm{DM}$ is of interest, as there is already considerable evidence that pre-existing DM contributes to the severity and mortality of COVID patients [30], and our data suggest that it may also contribute to the likelihood of survivors developing long-term pulmonary sequelae. Conversely, although premorbid obesity is well documented to increase severity and mortality in COVID patients [31, 32], in our cohort we found no association between BMI and persistent symptoms, nor did we find any relationship between BMI and percentage of predicted TLCO at follow-up. We also did not find any association between pre-admission inhaled corticosteroid use and persistent symptoms. This is unexpected in light of the recent STOIC study, which showed that COVID patients treated with inhaled corticosteroids in the community had decreased rates of hospital admission, and also a quicker resolution of selfreported and questionnaire-reported symptoms [33]. We speculate that the reasons we did not see similar results were partly due to the low numbers prescribed inhaled corticosteroids in our study, and also because of the different settings (real-world evidence compared with randomised control trial). Although we were able to obtain record of prescriptions, we had no means of measuring compliance. We speculate that compliance was likely to be lower in our patients, who were being treated for chronic conditions, compared to acutely unwell participants recruited to a COVID therapy trial.

In our ICU cohort, we observed a higher prevalence of TLCO abnormalities than the ward-based cohort, but this did not quite reach statistical significance. In the ICU cohort, the percentage of predicted TLCO correlated negatively with increased LOS and with higher maximum IPAP, but did not correlate with maximum inspired $\mathrm{FiO}_{2}$ (likely due to the high levels of $\mathrm{FiO}_{2}$ used in all these patients). In the entire patient group, the percentage of predicted TLCO correlated negatively with both increased LOS and higher maximum $\mathrm{FiO}_{2}$. Radiological signs of GGO and fibrosis showed a statistically significant increase in the ICU cohort compared to the ward-based cohort.

Our findings are in keeping with the few existing studies in the literature. Radiographic abnormalities have been reported in $18-94 \%$ of COVID patients at follow-up, and abnormalities of pulmonary function tests in $25-88 \%$ of these patients. One study of 18 patients reported radiological signs of fibrosis in $84 \%$ of their subjects [26], but others have reported such changes in between 7 and $26 \%$ of subjects, reflective of our findings above $[13,14,16,17,24]$. The most frequently reported PFT abnormality is an abnormal TLCO, reported in $16-72 \%$ of patients $[13,14,16$, $19,21,22,24,25,27]$. Very few studies have reported associations with persistent pathological CT findings and lung function abnormalities. Lerum et al. reported an association between ICU admission and pathological CT findings [14] in a cohort of 15 ICU patients, and Smet et al. reported an association between abnormalities in TLCO and increasing LOS, as well as with admission to ICU [22], all in keeping with our findings. Shah et al. reported an association between the number of days requiring supplemental oxygen and TLCO and CT abnormalities. Collectively, this allows us to start to build a picture of the patients who are at greater risk of long-term pulmonary sequelae after COVID infection, with LOS, oxygen requirements and ICU admission as useful 
indicators of severity of disease and increased risk of later problems.

A limitation of our study is the bias introduced by only performing PFTs and CT thorax on those with self-reported persistent symptoms at 8 weeks, or with persistent changes on chest radiograph. Furthermore, we did not have both PFTs and CTs for all patients intended for follow-up, due to delays in booking appointments, patients failing to attend, or inability to complete tests, again reflective of collecting data from a 'real world' follow-up setting. This very likely means that some cases of post-COVID morbidity are not captured. With this caveat, the prevalence of detected persistent pulmonary function test abnormalities within our entire cohort $(n=221)$ was $53(24 \%)$. The prevalence of detected persistent radiological interstitial changes post-COVID within our entire cohort $(n=221)$ was $50(23 \%)$. The prevalence of persistent fibrosis was 15 (7\%). However, in the absence of CT scans for every individual, these may not be truly representative figures. Nonetheless, in real-world settings, it would not be practical or ethical to offer CTs scans to all follow-up patients, and therefore this gives some estimate of the likely prevalence of symptomatic post-COVID fibrosis based on a more pragmatic approach that should be applicable to most settings.

Of further note, we do not yet know the long-term natural history of the radiological appearances, and it remains possible that there may be significant improvement further down the line. Indeed, one very recent study has reported that CT abnormalities continue to improve at 6-months post-infection, although PFT abnormalities, in particular restrictive defects, may persist [34].

An additional limitation of our study is the absence of pre-morbid PFTs and imaging in the majority of our patients, meaning that we cannot attribute with certainty the abnormalities seen to the damaging effects of COVID infection. This is reflected in the increased prevalence of obstructive and small airway defects in our non-ICU follow-up cohort as compared to our ICU cohort, which we speculate is likely explained by the increased prevalence of COPD and ex- and current smokers in this group.
Finally, our data report on follow-up from patients treated very early in the pandemic, when no medical interventions beyond best supportive care were in routine use for the treatment of COVID. Since then, advances have been made in the treatment of COVID-19, most notably with the introduction of dexamethasone for all hospitalised patients requiring supplemental oxygen, as well as the antiviral remdesivir and the anti-interleukin 6 monoclonal antibody tocilizumab. The advent of these treatments will very likely impact on the long-term consequences of COVID infection. The RECOVERY trial was introduced during the period of recruitment for this study, and a few of the patients in this study were recruited and randomised to this clinical trial (data not shown). It will be extremely interesting to discover whether any of these treatments also reduce long-term pulmonary sequelae in COVID survivors. However, we did not feel that our study was an appropriate platform to address this question, and felt this was better examined within the context of the clinical trial analysis.

Nonetheless, our study contributes to the emerging literature on post-COVID lung sequelae. Notably, we have one of the longest post-infection follow-up time of studies to date, with an average of 15 weeks from discharge to PFTs and 18 weeks from discharge to CT thorax. Of the existing published studies, there are only five reporting at 12 weeks or longer after infection $[14,16,21,24,27]$, and only one reporting beyond 3 months after discharge [33]. BTS do not recommend evaluation of PFTs prior to 3 months after discharge [28], as premature evaluation may lead to errors in functional diagnosis, and any abnormalities may still reflect the resolving acute inflammatory process rather than longer-term sequelae. In addition, to the best of our knowledge, we have presented the first description of experience of implementing the new BTS guidelines for COVID follow-up, and, as such, the data presented here can help inform and guide the development of equivalent services as we enter the post-COVID era. 


\section{CONCLUSION}

Our data add to the growing evidence that there will be pulmonary sequelae in a proportion of COVID survivors, providing some insight into what may become a significant chronic global health problem. We can offer the following estimate from our findings which others may find useful: services using BTS guidelines to guide follow-up of post-COVID hospitalised patients may see an overall prevalence of PFT abnormalities in around $24 \%$ and radiological fibrosis in around $7 \%$ at 4 months after discharge.

\section{ACKNOWLEDGEMENTS}

Funding. Rebecca Robey is an Academic Clinical Fellow funded by the National Institute of Health Research, UK. No funding or sponsorship was received for this study or publication of this article.

Authorship. All named authors meet the International Committee of Medical Journal Editors (ICMJE) criteria for authorship for this article, take responsibility for the integrity of the work as a whole, and have given the approval for this version to be published.

Author Contributions. Conceptualisation, Cristina Avram, Nazia Chaudhuri, Pilar RiveraOrtega, Veronica Yioe; Methodology, Cristina Avram, Nazia Chaudhuri, Katie Kemp, Philip Hayton, Rebecca Robey, Pilar Rivera-Ortega, Veronica Yioe; Formal Analysis, Cristina Avram, Nazia Chaudhuri, Melanie Greaves, Rebecca Robey, Ran Wang, Data Curation, Katie Kemp, Philip Hayton, Dalia Mudawi, Rebecca Robey, Ran Wang, Writing-Original Draft Preparation, Philip Hayton, Katie Kemp, Rebecca Robey, Writing-Review and Editing, Cristina Avram, Nazia Chaudhuri, Melanie Greaves, Katie Kemp, Philip Hayton, Dalia Mudawi, Rebecca Robey, Pilar Rivera-Ortega, Veronica Yioe, Ran Wang, Supervision, Cristina Avram, Nazia Chaudhuri.
Disclosures. Rebecca C. Robey, Katie Kemp, Philip Hayton, Dalia Mudawi, Ran Wang, Melanie Greaves, Veronica Yioe, Pilar RiveraOrtega, Cristina Avram, and Nazia Chaudhuri have nothing to disclose.

Compliance with Ethics Guidelines. The study was conducted according to the guidelines of the Declaration of Helsinki of 1964, and its later amendments. Ethical review and approval were waived for this study, due to the work conducted being part of local service evaluation, utilising only data routinely collected as part of the local COVID follow-up service. As such, specific ethical approval and informed patient consent were not required, as confirmed by the NHS Health Research Authority decision tool. This service evaluation is registered locally.

Data Availability. The datasets generated during and/or analyzed during the current study are available from the corresponding author on reasonable request.

Open Access. This article is licensed under a Creative Commons Attribution-NonCommercial 4.0 International License, which permits any non-commercial use, sharing, adaptation, distribution and reproduction in any medium or format, as long as you give appropriate credit to the original author(s) and the source, provide a link to the Creative Commons licence, and indicate if changes were made. The images or other third party material in this article are included in the article's Creative Commons licence, unless indicated otherwise in a credit line to the material. If material is not included in the article's Creative Commons licence and your intended use is not permitted by statutory regulation or exceeds the permitted use, you will need to obtain permission directly from the copyright holder. To view a copy of this licence, visit http:// creativecommons.org/licenses/by-nc/4.0/. 


\section{REFERENCES}

1. https://www.who.int/csr/don/05-january-2020pneumonia-of-unkown-cause-china/en/. Data extracted 30th December 2020.

2. https://covid19.who.int. Data extracted 30th December 2020.

3. Torres-Castro R, Vasconcello-Castillo L, AlsinaRestoy X, Solis-Navarro L, Burgos F, Puppo H, Vilaró $\mathrm{J}$. Respiratory function in patients post-infection by COVID-19: a systematic review and meta-analysis. Pulmonology. 2020;S2531-0437(20):30245-52.

4. Guan WJ, Ni ZY, Liang WH, Ou CQ, He JX, Liu L, Shan H, Lei CL, Hui DSC, Du B, Li LJ, for the China Medical Treatment Expert Group for Covid-19, et al. Clinical characteristics of coronavirus disease 2019 in China. NEJM. 2020;382:1708-20.

5. Hui DS, Wong KT, Ko FW, Tam LS, Chan DP, Woo J, Sung JJ. The 1-year impact of severe acute respiratory syndrome on pulmonary function, exercise capacity, and quality of life in a cohort of survivors. Chest. 2005;128(4):2247-61.

6. Das KM, Lee EY, Singh R, Enani MA, Al Dossari K, Van Gorkom K, Larsson SG, Langer RD. Follow-up chest radiographic findings in patients with MERS$\mathrm{CoV}$ after recovery. Indian $\mathrm{J}$ Radiol Imaging. 2017;27(3):342-9.

7. Fumagalli A, Misuraca C, Bianchi A, Borsa N, Limonta S, Maggiolini S, Bonardi DR, Corsonello A, Di Rosa M, Soraci L, Lattanzio F, Colombo D. Pulmonary function in patients surviving to COVID-19 pneumonia. Infection. 2020;28:1-5.

8. Picchi G, Mari A, Ricciardi A, Carucci AC, Sinatti G, Cosimini B, Di Norcia M, Iapadre N, Balsano C, Grimaldi A. Three cases of COVID-19 pneumonia in female patients in Italy who had pulmonary fibrosis on follow-up lung computed tomography imaging. Am J Case Rep. 2020;21:e926921.

9. Scelfo C, Fontana M, Casalini E, Menzella F, Piro R, Zerbini A, Spaggiari L, Ghidorsi L, Ghidoni G, Facciolongo NC. A dangerous consequence of the recent pandemic: early lung fibrosis following COVID-19 pneumonia-case reports. Ther Clin Risk Manag. 2020;29(16):1039-46.

10. Zha L, Shen Y, Pan L, Han M, Yang G, Teng X, Tefsen B. Follow-up study on pulmonary function and radiological changes in critically ill patients with COVID-19. J Infect. 2020;S0163-4453(20): 30317.

11. Daher A, Balfanz P, Cornelissen C, Müller A, Bergs I, Marx N, Müller-Wieland D, Hartmann B, Dreher M,
Müller T. Follow up of patients with severe coronavirus disease 2019 (COVID-19): pulmonary and extrapulmonary disease sequelae. Respir Med. 2020;174:106197.

12. Frija-Masson J, Debray MP, Gilbert M, Lescure FX, Travert F, Borie R, Khalil A, Crestani B, d'Ortho MP, Bancal C. Functional characteristics of patients with SARS-CoV-2 pneumonia at 30 days post-infection. Eur Respir J. 2020;56(2):2001754.

13. Huang $Y$, Tan $C$, Wu J, Chen M, Wang Z, Luo L, Zhou X, Liu X, Huang X, Yuan S, Chen C, Gao F, Huang J, Shan H, Liu J. Impact of coronavirus disease 2019 on pulmonary function in early convalescence phase. Respir Res. 2020;21(1):163.

14. Lerum TV, Aaløkken TM, Brønstad E, Aarli B, Ikdahl E, Lund KMA, Durheim MT, Rodriguez JR, Meltzer C, Tonby K, Stavem K, Skjønsberg OH, Ashraf H, Einvik G. Dyspnoea, lung function and CT findings three months after hospital admission for COVID19. Eur Respir J. 2020;10:2003448.

15. Li X, Wang C, Kou S, Luo P, Zhao M, Yu K. Lung ventilation function characteristics of survivors from severe COVID-19: a prospective study. Crit Care. 2020;24(1):300.

16. Liang L, Yang B, Jiang N, Fu W, He X, Zhou Y, Ma WL, Wang $X$. Three-month follow-up study of survivors of coronavirus disease 2019 after discharge. J Korean Med Sci. 2020;35(47):e418.

17. Liu D, Zhang W, Pan F, Li L, Yang L, Zheng D, Wang J, Liang B. The pulmonary sequalae in discharged patients with COVID-19: a short-term observational study. Respir Res. 2020;21(1):125.

18. Liu K, Zhang W, Yang Y, Zhang J, Li Y, Chen Y. Respiratory rehabilitation in elderly patients with COVID-19: a randomized controlled study. Complement Ther Clin Pract. 2020;39:101166.

19. Mo X, Jian W, Su Z, Chen M, Peng H, Peng P, Lei C, Chen R, Zhong N, Li S. Abnormal pulmonary function in COVID-19 patients at time of hospital discharge. Eur Respir J. 2020;55(6):2001217.

20. Rogliani P, Calzetta L, Coppola A, Puxeddu E, Sergiacomi G, D'Amato D, Orlacchio A. Are there pulmonary sequelae in patients recovering from COVID-19? Respir Res. 2020;21(1):286.

21. Shah AS, Wong AW, Hague CJ, Murphy DT, Johnston JC, Ryerson CJ, Carlsten C. A prospective study of 12-week respiratory outcomes in COVID-19-related hospitalisations. Thorax. 2020. https://doi. org/10.1136/thoraxjnl-2020-216308.

22. Smet J, Stylemans D, Hanon S, Ilsen B, Verbanck S, Vanderhelst E. Clinical status and lung function 10 
weeks after severe SARS-CoV-2 infection. Respir Med. 2020;176:106276.

23. Trinkmann F, Müller M, Reif A, Kahn N, Kreuter M, Trudzinski F, Eichinger M, Heussel CP, Herth FJF, Lung Network Rhine-Neckar-Region. Residual symptoms and lower lung function in patients recovering from SARS-CoV-2 infection. Eur Respir J. 2021;57:2003002.

24. van den Borst B, Peters JB, Brink M, Schoon Y, Bleeker-Rovers CP, Schers $\mathrm{H}$, van Hees $\mathrm{HWH}$, van Helvoort $H$, van den Boogaard $M$, van der Hoeven H, Reijers $\mathrm{MH}$, Prokop M, Vercoulen J, van den Heuvel M. Comprehensive health assessment three months after recovery from acute COVID-19. Clin Infect Dis. 2020. https://doi.org/10.1093/cid/ ciaa 1750.

25. van der Sar-van der Brugge $S$, Talman $S$, Boonmande Winter L, de Mol M, Hoefman E, van Etten RW, De Backer IC. Pulmonary function and health-related quality of life after COVID-19 pneumonia. Respir Med. 2020;176:106272.

26. You J, Zhang L, Ni-Jia-Ti MY, Zhang J, Hu F, Chen L, Dong Y, Yang K, Zhang B, Zhang S. Anormal pulmonary function and residual CT abnormalities in rehabilitating COVID-19 patients after discharge. J Infect. 2020;81(2):e150-2.

27. Zhao YM, Shang YM, Song WB, Li QQ, Xie H, Xu QF, Jia JL, Li LM, Mao HL, Zhou XM, Luo H, Gao YF, $\mathrm{Xu}$ AG. Follow-up study of the pulmonary function and related physiological characteristics of COVID19 survivors three months after recovery. EClinicalMedicine. 2020;25:100463.

28. British Thoracic society guidance on respiratory follow up of patients with a clinico-radiological diagnosis of COVID-19 pneumonia. Available from: https://www.brit-thoracic.org.uk/documentlibrary/quality-improvement/covid-19/resp-followup-guidance-post-covid-pneumonia/.

29. Hare SS, Rodrigues JCL, Nair A, Jacob J, Upile S, Johnstone A, Mcstay R, Edey A, Robinson G. The continuing evolution of COVID-19 imaging pathways in the UK: a British Society of Thoracic Imaging expert reference group update. Clin Radiol. 2020;75:399-404.

30. Abdi A, Jalilian M, Sarbarzeh PA, Vlaisavljevic Z. Diabetes and COVID-19: a systematic review on the current evidences. Diabetes Res Clin Pract. 2020;166:108347.

31. Hussain A, Mahawar K, Xia Z, Yang W, El-Hasani S. Obesity and mortality of COVID-19. Meta-analysis. Obes Res Clin Pract. 2020;14(4):295-300.

32. Yang J, Hu J, Zhu C. Obesity aggravates COVID-19: a systematic review and meta-analysis. J Med Virol. 2020. https://doi.org/10.1002/jmv.26237.

33. Ramakrishnan S, Nicolau DV Jr, Langford B, Mahdi M, Jeffers H, Mwasuku C, Krassowska K, Fox R, Binnian I, Glover V, Bright S, Butler C, Cane JL, Halner A, Matthews PC, Donnelly LE, Simpson JL, Baker JR, Fadai NT, Peterson S, Bengtsson T, Barnes PJ, Russell REK, Bafadhel M. Inhaled budesonide in the treatment of early COVID-19 (STOIC): a phase 2, open-label, randomised controlled trial. Lancet Respir Med. 2021. (Epub ahead of print).

34. Stylemans, D, Smet J, Hanon, S, Schuermans D, Ilsen B, Vandemeulebroucke J, Vanderhelst E, Verbanck S. Evolution of lung function and chest CT 6 months after COVID-19 pneumonia: real-life data from a Belgian University Hospital. Respir Med. 2021. (Epub ahead of print). 